\title{
Improviser en classe de Français Langue Étrangère : spontanéité et réflexion à travers le théâtre-forum
}

\author{
Claire Del Olmo \\ Université de Toulouse 2 - Jean-Jaurès \\ France
}

\section{Introduction}

Cela fait plus d'un demi-siècle que les didacticiens spécialisés dans l'enseignement des Langues Étrangères (LE) mettent en lumière les apports des interactions simulées pour l'apprentissage de la langue cible. L'entrée du «je » de l'apprenant dans le «jeu » de la communication est souvent préconisée pour développer les compétences dans la LE. Aujourd'hui, les enseignants invitent les apprenants à entrer dans des « jeux » où les actions du « je » sont improvisées. Le « je » de l'apprenant trouve un espace où s'exprimer sans frontière.

\section{Des simulations «programmées » aux simulations « improvisées »}

Durant les années 1960, de nombreux enseignants de LE recourent à la méthodologie Structuro-Globale Audio-Visuelle (SGAV). Leurs séances pédagogiques suivent généralement trois grandes étapes (Robert 202). Quand un enseignant adopte cette méthodologie, il présente d'abord à ses apprenants un dialogue via une bande son, en leur projetant une bande image pour illustrer la situation de communication. II les invite, ensuite, à participer à une série d'activités pour qu'ils s'approprient le dialogue. Une fois le dialogue appris, les apprenants réalisent un jeu de rôles qui suit de près son intrigue. Ils ont des rôles correspondant à ceux des personnages et sont ainsi amenés à formuler des énoncés en accord avec les actes de langage du dialogue présenté en début de séance (Leblanc 16). Le jeu de rôles permet surtout d'amener les apprenants à réemployer les structures langagières qu'ils ont mémorisées.

Les enseignants qui s'inscrivent, plus tard, dans les Approches Communicatives (AC), nées pendant les années 1980, continuent à proposer aux apprenants de participer à des jeux de rôles. La simulation est l'activité de référence des AC (Puren 37). Elle permet aux apprenants de jouer des rôles d'usagers dans la société dont la langue est apprise (Puren 37). Avec ce type d'activité, les apprenants doivent mettre en œuvre leurs savoirs et savoir-faire linguistiques ainsi que socioculturels, comme s'ils étaient en situation de communication réelle, afin de réaliser un scénario préalablement défini (Cuq 221). De manière générale, pour préparer une simulation, ils participent à des activités portant sur des documents authentiques. Celles-ci sont cognitives avec des tâches de perception d'information, d'anticipation du contenu, de repérage, de conceptualisation et elles permettent, par ailleurs, la systématisation et le réemploi avec des tâches de répétition et d'expression (Lemeunier 355). Grâce à ces tâches, les apprenants acquièrent des structures langagières qu'ils réemploient via des jeux de rôles.

Les jeux de rôles des AC sont ainsi relativement proches de ceux effectués dans le cadre de la méthodologie SGAV. Ils ont le même but : permettre aux apprenants de réaliser certains énoncés de façon privilégiée grâce à des scénarios bien établis. Or il convient de noter, à ce propos, que la pertinence de leur apport est questionnée par les didacticiens. Selon Valérie Lemeunier, la faible créativité des apprenants dans les jeux de rôles les conduirait à éprouver des difficultés lorsqu'ils sont en situation de communication réelle avec des natifs (362). En effet, il est possible qu'un apprenant ne parvienne pas à réemployer les formes langagières acquises au sein de l'espace classe présentées dans un cadre de communication extrêmement spécifique. II risque d'être souvent « frustré et paniqué dès qu'il doit communiquer hors du modèle » (Lemeunier 362).

Face aux limites de l'apprentissage d'une LE via les jeux de rôles, il n'est pas étonnant que les enseignants de FLE se tournent vers d'autres formes de « jeux ». Aujourd'hui, les formateurs laissent de plus en plus de place à l'imagination des apprenants dans le « jeu » de la communication. Ils leur proposent des « jeux » qui leur permettent une pratique plus libre de la langue cible. Ils recourent à l'improvisation théâtrale. Les didacticiens soulignent, eux, l'intérêt particulier du théâtre-forum. 


\section{Le théâtre-forum : improviser pour changer le monde}

Avant de préciser l'intérêt de recourir au théâtre-forum pour enseigner le FLE, expliquons ses origines ; cela nous permettra d'en cerner davantage les apports dans le champ qui nous intéresse. Le théâtre-forum est une technique théâtrale interactive créée dans les années 1960 par Augusto Boal au Brésil dans le but de résister culturellement à la dictature brésilienne. Ce sont les réactions des spectateurs, lors de représentations d'AGITPROP (Agitation et propagande) données par Boal et sa troupe (La compagnie du Théâtre de l'Opprimé), reprenant des formes de théâtre provenant de Russie et ayant pour but d'amener la classe prolétaire à prendre conscience de son oppression et à se révolter, qui conduisent le dramaturge à créer le théâtre-forum tel qu'on le connaît aujourd'hui.

C'est tout d'abord la réaction d'un spectateur nommé Virgilio qui conduit Boal à créer les premiers contours du théâtre-forum (Ramat 40). Suite à un spectacle où la troupe de Boal finit par encourager les spectateurs à s'engager dans la lutte armée pour s'approprier les terres, Virgilio explique aux acteurs qu'il est déjà engagé dans la lutte parce qu'il occupe, avec d'autres, les terres d'un grand propriétaire. Virgilio demande à Boal et sa troupe de le rejoindre dans la lutte. Les acteurs se rendent ainsi compte du paradoxe de leur œuvre. II est, pour eux, impossible d'inciter les spectateurs à réaliser des actions auxquelles ils ne se joindraient pas.

De fait, Boal se dirige vers une nouvelle forme de théâtre. Sa troupe en vient à présenter des situations problématiques aux spectateurs en les invitant à réfléchir aux actions qu'ils souhaiteraient mener pour les changer. En général, avant de donner une représentation, les acteurs mènent une enquête sur les expériences sociales et politiques qui posent problème aux spectateurs. Le lendemain, ils présentent sur scène ces situations complexes sur le plan politico-social, comme celle par exemple d'un adultère mettant en avant la problématique du statut de divorcé. Les spectateurs leur donnent des conseils pour que la situation exposée puisse changer.

C'est alors que la réaction d'une autre spectatrice conduit Boal à conceptualiser la relation entre le spectateur et l'acteur propre au théâtre-forum (Ramat 41). Lorsque la troupe de Boal joue précisément la scène d'une femme trompée, des spectateurs expliquent qu'avoir le statut de femme divorcée n'est pas accepté dans les années 1960 au Brésil. Une spectatrice propose ainsi que le couple ait une sérieuse discussion puis reprenne le cours de sa vie. Les acteurs jouent cette discussion plusieurs fois. Cependant, selon la spectatrice, ils ne suivent jamais ses conseils. Boal finit par inviter la spectatrice à venir sur scène pour qu'elle montre, ellemême, comment apporter une solution à cette situation. La spectatrice prend alors un balai et bat l'acteur jouant le mari. Puis, elle précise que suite à cette sérieuse discussion, ils peuvent reprendre le cours de leur vie. Dès lors, le rapport spectateur-acteur spécifique au théâtre forum est né.

Dans le théâtre-forum, les « spect-acteurs » pensent le monde grâce au langage théâtral et pas seulement au verbe (Boal, The Aesthetics of the Oppressed 6 ; notre traduction). Ils sont « cocréateurs et coacteurs de scénarios improvisés » (d'Orgeval 677). Le Théâtre de l'Opprimé s'éloigne des formes traditionnelles du théâtre puisque cet art est considéré « comme langage (qui doit être parlé) et non comme discours (qui doit être entendu), comme processus (qui doit être développé) non comme produit achevé (qui doit être consommé) parce qu'il propose l'opprimé comme sujet et non comme objet » (Boal, « Le flic dans la tête ou les trois hypothèses »165).

Le but du théâtre-forum est d'avoir un impact sur la vie sociale. Un spectacle basé sur cette technique de théâtre n'est pas une fin en soi. Au contraire, « la fin est le commencement » (Boal, The Aesthetics of the Oppressed 6). Le Théâtre de l'Opprimé, en plus d'être un outil qui conduit à mieux comprendre la réalité, permet un changement de l'environnement du spect'acteur (Boal, The Aesthetics of the Oppressed 7). II met en scène les rapports de domination afin de « permettre progressivement leur dévoilement, par un travail de conscience pour repousser les limites, les effets de clôtures, et, ainsi d'augmenter le répertoire possible de résistance et de transformation » (Bordet 161). II a un effet sur une situation que le spect-acteur vit ou vivra plus tard. Pour Boal, le théâtre-forum permet la « construction d'un modèle d'action futur [. . .] le spectateur, par l'acte ou la conduite qu'il aura adopté pendant une séance de théâtre forum, est en réalité incité à le reproduire dans la vie courante par la prise de conscience que cet acte ou cette conduite auront éveillé chez lui » (Evers 164). 
Habituellement, les acteurs d'un spectacle de théâtre-forum jouent une situation posant problème aux spectateurs. Puis, les spect-acteurs peuvent intervenir pour en changer le dénouement. Pour cela, ils remplacent les personnages. Ils s'essayent ensemble à «l'action transformatrice » et évaluent ses conséquences (Ramat, 2011 : 37). C'est le joker, un individu désigné, ayant le rôle de « meneur de jeu » et assurant le bon déroulement d'une séance (Guerre 86), qui repère les participants qui souhaitent intervenir et leur donne la parole.

\section{Les principes et apports du théâtre-forum dans le champ du FLE}

Aujourd'hui, les enseignants de FLE, qui travaillent à l'Université, recourent souvent au théâtre-forum. Ils y voient le moyen de dépasser les limites des jeux de rôles avec leurs scénarios préétablis en ce qui concerne l'enseignement/apprentissage de la langue cible.

Jacqueline Breugnot souligne l'intérêt que présente l'improvisation inhérente au théâtre-forum pour enseigner le FLE. Pour elle, ce type d'improvisation a essentiellement un avantage :

\section{Communicatif}

Elle conduit les apprenants à développer leurs stratégies de communication verbales et non verbales. Le théâtre-forum a pour objectif la résolution d'un problème et « pour y parvenir, les apprenants devront activer toutes leurs connaissances linguistiques, culturelles et stratégiques » (Breugnot, «La compréhension interculturelle en zone frontalière » 102) ${ }^{1}$. L'improvisation, qu'ils doivent réaliser dans le cadre du théâtreforum pour régler une situation problématique, les amène à prendre des risques dans la langue cible et leur permet de se familiariser avec une prise de parole spontanée tout en développant leur capacité à mettre en place des stratégies de communication.

\section{Didactique}

Elle conduit les apprenants à s'impliquer affectivement et leurs émotions leur permettent de mieux mémoriser les formes verbales et non verbales de la langue cible qu'ils perçoivent (Breugnot, «La formation interculturelle face à la diversité culturelle » 4) ;

\section{Cognitif}

Elle facilite la connexion entre les informations nouvelles et les connaissances déjà acquises (Breugnot, « La formation interculturelle face à la diversité culturelle »4) ;

\section{Interculturel}

Elle permet aux apprenants de voir comment une situation est envisageable sous différents angles. Ceux qui ont vécu des situations de manière négative auront tendance à ne pas généraliser leurs jugements sur le monde cible (Breugnot, « La formation interculturelle face à la diversité culturelle » 5). Souvent, les apprenants montrent dans les jeux du théâtre-forum des situations de domination sous différents aspects et le fait que ces situations soient prises en compte et que les apprenants y réfléchissent au sein d'un atelier permet d'avoir un effet sur les «perceptions de type paranoïaque » qu'ils peuvent développer après une expérience malheureuse (Breugnot, « La formation interculturelle face à la diversité culturelle » 5).

De fait, l'improvisation constitutive du théâtre-forum représente tant un moyen de mettre en place des interactions sans scénario préconçu que de favoriser la mémorisation de structures relatives à la langue cible ou de préparer à la rencontre interculturelle. II n'est donc pas rare que dans le domaine de l'enseignement des LE, le théâtre-forum soit envisagé comme un moyen privilégié pour développer la compétence de communication interculturelle.

Anne Pauzet rapporte, d'ailleurs, comment une expérience de théâtre-forum menée à l'Université d'Angers conduit des apprenants de FLE à dépasser les malentendus, incompréhensions ou chocs culturels qu'ils peuvent éprouver en situations de communication authentiques avec des natifs.

Dans les séances de théâtre-forum qui ont été mises en place, des apprenants ont dû créer des saynètes autour d'un problème interculturel. Ils ont été invités à suivre la consigne suivante: « Vous allez jouer une petite scène qui aura un début, un milieu et une fin. Ce ne sont pas des idées en général, ce n'est pas une 
idée sur les Français, c'est quelque chose que vous vivez dans votre vie de tous les jours et que vous aimeriez changer » (Pauzet 3). Les apprenants devaient préparer une scène montrant une interprétation du monde qu'ils n'arrivaient pas à accepter et qu'ils auraient aimé voir évoluer.

Puis, lorsque des apprenants présentaient une saynète, une question était posée à l'ensemble du groupe. Par exemple, si était exposé un mal-être résultant de normes proxémiques différentes dans la culture de l'apprenant et la culture étrangère, la question posée aux participants était : « Comment faire pour respecter la distance de l'autre ? » (Pauzet 3). Les apprenants qui ont tenté de répondre à cette question, et ont pour cela remplacé l'un des protagonistes pour changer la situation montrée, ont été amenés à réfléchir aux cultures représentées et à leur propre culture. C'est pourquoi le théâtre-forum permet de développer une « meilleure connaissance des autres (comportements, croyances, représentations, valeurs...) et, en retour, une meilleure connaissance de soi et ainsi de s'exercer à la relativité » (Pauzet 4). Il accroît la compétence de communication interculturelle.

Dans le théâtre-forum, l'improvisation d'une solution à un problème interculturel nécessite préalablement et de façon continue le dialogue entre le « je » de l'apprenant et l'Autre avec ses manières de faire et ses façons de penser. La réflexion permettant d'improviser une solution à une situation interculturelle problématique amène les apprenants à relativiser des comportements qui les gênent. A travers la rencontre de l'Autre via leur subjectivité à l'œuvre dans l'improvisation, ils se préparent à une rencontre interculturelle authentique.

\section{Retour d'expérience}

Nous avons, de notre côté, mis en place quelques séances de théâtre-forum en 2012, auprès d'étudiants Erasmus de l'Université de Toulouse 2 Jean Jaurès dans le cadre d'un cours de FLE. Cela nous a permis d'observer l'intérêt de l'improvisation intrinsèque à cette forme de théâtre. Les étudiants Erasmus qui ont participé à ces séances avaient acquis un niveau B1 en français. Les ateliers de théâtre-forum ont eu lieu au printemps et ont été menés en extérieur.

Voici comment, étape par étape, nous avons intégré cette technique à nos classes. Tout d'abord, dans nos séances, les apprenants devaient constituer des binômes. Nous leur accordions une période de 15 minutes pour inventer et mettre en scène une situation conflictuelle qui finissait mal. Puis, nous passions de groupe en groupe pour apporter un soutien linguistique et phonétique, voire culturel si nécessaire. Ensuite venait le moment pour chaque binôme de présenter sa saynète. Après chaque intervention, nous laissions quelques minutes pour la réflexion collective en demandant aux apprenants de décrire la situation montrée et de mettre en avant le problème qu'ils identifiaient. Une fois le problème cerné, le binôme rejouait une seconde fois sa saynète. Les autres apprenants pouvaient alors changer le cours de la situation en remplaçant l'un des personnages et en improvisant, en situation, une parole gesticulée. Pour cela, les apprenants-spect'acteurs devaient taper des mains. Lorsque l'un d'eux tapait des mains, le binôme se figeait. L'apprenant-spec'acteur souhaitant intervenir pour changer la situation remplaçait l'un des personnages. Puis, la scène reprenait là où elle avait été arrêtée. Ensuite, un autre apprenant-spect'acteur pouvait frapper des mains et remplacer un personnage pour tenter de changer le cours des choses lorsqu'il le souhaitait. Lorsqu'un apprenant spect'acteur parvenait à proposer une solution satisfaisante pour tous, nous réalisions un dernier échange sur la solution mise en avant.

Avec ces ateliers, nous avons pu observer plusieurs sortes d'impact de l'improvisation du «je » des apprenants dans le « jeu » du théâtre-forum sur l'apprentissage :

Incitation à la prise de risque

Tous les participants, qu'ils soient timides ou extravertis, intervenaient dans le théâtre-forum en tant que spect'acteur. Tous proposaient, via l'improvisation d'une parole dans un corps en mouvements, des solutions aux situations problématiques exposées.

\section{Attention}

Lorsque les apprenants devaient se risquer à des prises de parole improvisées pour changer les situations montrées dans le théâtre-forum, ils étaient très attentifs. Nous avons observé une corrélation entre les activités d'improvisation et l'augmentation de l'attention des apprenants. 


\section{Motivation}

Les participants semblaient, de plus, très intéressés par les solutions improvisées par les apprenants-spectacteurs. Ces improvisations suscitaient aussi chez eux de nombreux rires. Les participants semblaient ainsi avoir une motivation intrinsèque importante.

\section{Cohésion}

Les improvisations des apprenants-spect-acteurs, s'insérant dans des situations pour échanger avec d'autres et tenter de trouver des solutions, ont nécessité et renforcé la confiance que les apprenants éprouvaient les uns envers les autres. Leur participation à des interactions collectives improvisées a développé la cohésion sociale du groupe-classe.

\section{Prononciation}

L'improvisation des apprenants-spect'acteurs leur a enfin permis de vivre une parole en mouvements. Elle a constitué pour eux un moyen d'entrer dans la corporalité de la LE de façon spontanée et de prononcer, en outre, la langue cible en bougeant à la manière des natifs.

Nous avons ainsi constaté plusieurs effets bénéfiques de l'improvisation du théâtre-forum pour l'enseignement/apprentissage du FLE. Grâce à elle, les apprenants sont incités à prendre des risques dans la langue cible. Elle les conduit aussi à être plus attentifs et les motive. L'improvisation augmente, par ailleurs, la cohésion du groupe-classe. Elle permet aussi aux apprenants de vivre la langue dans sa multidimensionnalité.

\section{Modélisation}

En considérant l'impact de l'improvisation du théâtre-forum sur l'apprentissage du FLE, mis en avant par les didacticiens et observé dans nos pratiques d'enseignement, nous pouvons proposer une modélisation. Avec celle-ci, nous souhaitons mettre en lumière la dynamique et les enjeux des apports de cette improvisation dans le cadre de l'apprentissage du FLE. La figure 1 présente notre modèle.

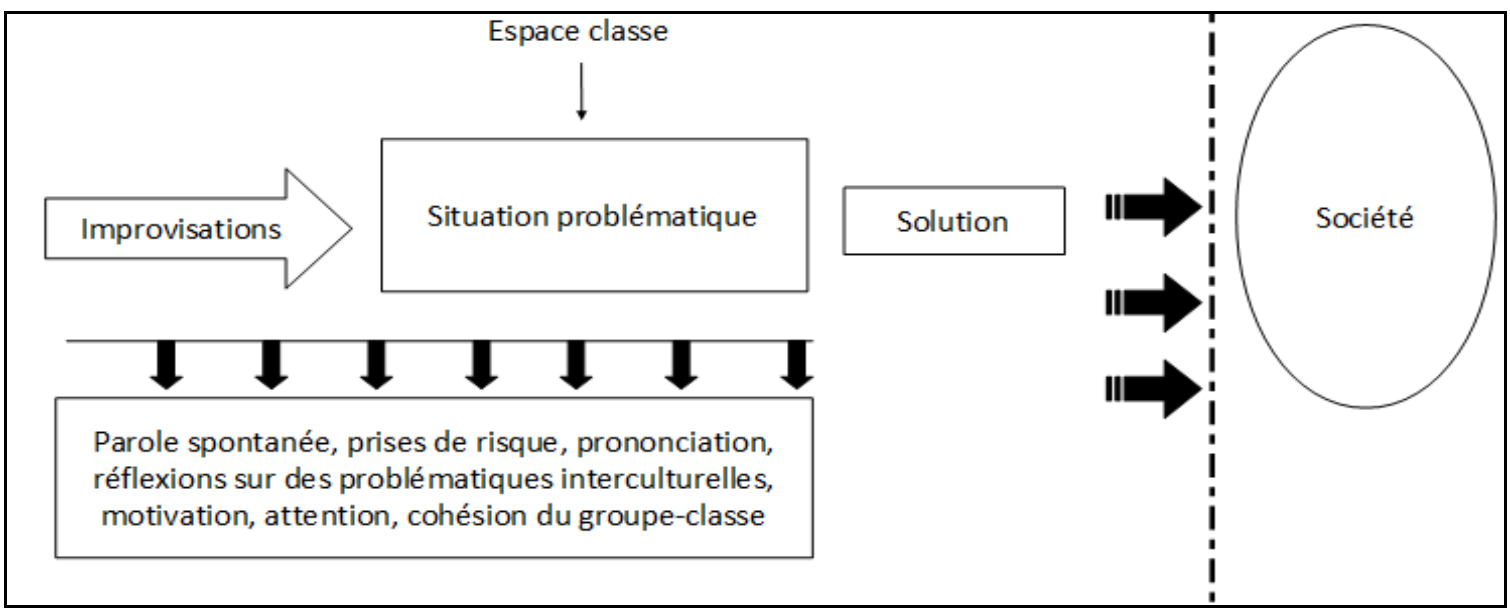

Figure 1 : Dynamique et enjeux de l'improvisation du théâtre-forum en FLE

Ainsi que l'indique la figure 1, le théâtre-forum a pour origine l'insertion d'une situation problématique au sein de l'espace classe. II est possible que cette situation, tel que nous l'avons précisé en amont, soit proposée par les apprenants eux-mêmes selon les consignes que leur fournit l'enseignant.

Une fois que des apprenants-acteurs ont inséré au sein de la classe une situation problématique en la jouant et que celle-ci en vient à constituer le cœur des réflexions du groupe-classe, les apprenants spect'acteurs se trouvent alors dans une situation de résolution de problème. Ils trouveront une solution à la scène exposée en remplaçant l'un des personnages. Comme le montre la figure 1, c'est par l'improvisation de comportements 
verbaux et non verbaux qu'ils vont entrer dans la situation montrée et viser son changement dans le but de dépasser les problèmes qu'elle présente.

Le fait que les apprenants-spect'acteurs improvisent le discours d'un personnage et ses actions pour modifier le contenu de la scène jouée a plusieurs effets sur leur apprentissage. Dans la figure 1, nous pouvons constater que l'improvisation leur permet d'abord de produire une parole spontanée et de prendre des risques dans la langue cible tout en ayant l'occasion d'entrer dans la corporalité inhérente à cette langue. L'improvisation représente aussi pour eux l'occasion de réfléchir à des problématiques interculturelles. Par ailleurs, elle les motive intrinsèquement et focalise, en outre, leur attention sur ce qui est joué. Ce qui devrait, d'ailleurs, les conduire à mieux mémoriser les informations verbales et non verbales qu'ils perçoivent. Enfin, l'improvisation permet d'accroître la cohésion du groupe-classe, condition qui est, notons-le, propice aux apprentissages réalisés en groupes au sein de la classe de FLE.

Finalement, tel que l'atteste la figure 1, l'improvisation des apprenants-spect'acteurs, pour apporter une solution au problème mis en avant lors d'une séance de théâtre-forum, aura toujours un effet, a postériori, dans la réalité de la société. Tout d'abord, les apprenants développent leur compétence communicative ; ce qui aura bien évidemment un impact sur leurs futures interactions authentiques avec des locuteurs de la langue cible. De plus, comme l'improvisation accroît la cohésion sociale et que les apprenants développent leur sentiment d'appartenance au groupe-classe, ces derniers pourront davantage mettre en place des stratégies pour faire appel aux membres du groupe afin d'optimiser leur apprentissage de la LE en dehors de l'espace classe. Puis, comme les apprenants-spect'acteurs peuvent être amenés à réfléchir à des situations comprenant des incompréhensions interculturelles, en devant improviser des comportements pour y remédier, il est probable qu'ils réagissent différemment lorsqu'ils rencontreront dans la réalité des situations constituées de problématiques similaires.

\section{Comment proposer un théâtre-forum pour une parole improvisée ?}

Comme nous venons de le souligner, l'improvisation du théâtre-forum est propice à l'apprentissage du FLE. L'enseignant de FLE qui souhaite bénéficier de ses apports a ainsi la possibilité de mettre en place un théâtreforum. Pour cela, il doit insérer au sein de l'espace classe une situation problématique, en proposant par exemple aux apprenants de réfléchir à un vécu interculturel qui leur pose problème comme le fait Pauzet (2010). L'enseignant a, par ailleurs, la possibilité d'utiliser d'autres techniques pour faire entrer ce type de situation au sein de la classe.

Selon nous, il existe un moyen très simple d'introduire au sein de l'espace classe la situation qui sera au centre des réflexions d'une séance de théâtre-forum. Le procédé le plus facile est de recourir au support filmique. Projeter un extrait de film permet à l'enseignant d'exposer aux apprenants la situation problématique qu'ils devront solutionner dans le cadre d'un atelier de théâtre-forum. Certes, lorsque l'enseignant sélectionne son support, il doit être attentif à son contenu. II faut que son extrait présente une scène problématique ou conflictuelle qui ne soit pas ou qui soit mal résolue.

L'enseignant désirant mettre en place un théâtre-forum à partir d'un extrait de film pourrait, en s'inspirant de Boal (Jeux pour acteurs et non-acteurs 59-61), créer une séance comprenant les étapes suivantes :

\section{1) Présentation de l'extrait}

L'enseignant projette aux apprenants un extrait et les invite à réaliser des activités de compréhension globale et détaillée.

2) Introduction du théâtre-forum

a) Réflexion générale sur l'extrait

II demande aux apprenants si la fin de l'extrait leur semble acceptable.

b) Présentation du théâtre-forum

L'enseignant joue le rôle de joker (dont la fonction est décrite plus haut) et présente l'activité. II précise que deux apprenants (dans le cas où l'extrait comprend deux personnages) vont rejouer la scène de l'extrait et que va alors commencer une « lutte-jeu » (Boal, Jeux pour acteurs et non-acteurs 59). II demande aux apprenants qui sont volontaires pour rejouer l'extrait de tenter de finir la scène de la même manière que dans l'extrait pendant toute la séance. II donne d'autres consignes au groupe. Les autres apprenants vont devoir modifier la 
fin de la scène en apportant des solutions qu'ils pourraient proposer dans une situation réelle. Ils pourront changer la situation en remplaçant le protagoniste qui essaie d'apporter une solution. Pour cela, ils devront s'approcher du lieu où les deux apprenants volontaires jouent la scène de l'extrait en criant « stop » et remplacer l'apprenant-acteur essayant de solutionner la situation pour la rejouer à partir du moment où ils l'auront arrêtée.

\section{3) Déroulement}

a) Première intervention

Une fois que les deux apprenants volontaires ont joué la scène de l'extrait, un premier apprenant-spect'acteur est autorisé à entrer dans le « jeu » pour remplacer le protagoniste qui essaie d'apporter une solution, en criant «stop ». C'est en improvisant pour remplacer ce personnage qu'il va essayer de changer le cours des événements.

b) Nouvelle intervention

Quand le premier apprenant-spect'ateur ne semble plus dans la capacité de faire évoluer la situation avec son improvisation, un autre apprenant-spect'acteur peut s'approcher du lieu où se joue la scène et crier « stop ». II remplace alors le protagoniste tentant de résoudre le problème exposé. II improvise une parole en mouvements pour expérimenter une nouvelle manière de dépasser la situation problématique de l'extrait.

\section{4) Clôture}

Lorsque les apprenants ne proposent plus d'improvisation pour apporter des solutions à la situation travaillée au sein de la classe, le joker se doit de proposer une discussion réflexive sur les solutions avancées pendant la séance. II convient ici de faire une remarque. Il est très important que le joker garde à l'esprit que sa fonction n'est pas de présenter le moyen du changement. Ce n'est pas « un conférencier, il n'est pas le détenteur de la vérité : tout juste essaiera-t-il de faire que ceux qui savent un peu plus l'expliquent ; que ceux qui osent un peu plus osent, montrent ce dont ils sont capables » (Boal, Jeux pour acteurs et non acteurs 61).

\section{Conclusion}

Avec cet article, nous mettons en lumière que l'improvisation du « je » de l'apprenant de FLE dans le cadre de séances de théâtre-forum est particulièrement propice à l'apprentissage de la langue cible. Cette improvisation, pouvant être réalisée au sein d'ateliers de deux heures par exemple, permet le développement de la compétence communicative, une amélioration de la prononciation ou encore l'accroissement de la motivation, de l'attention et de la cohésion du groupe-classe. Les pratiques du théâtre-forum dans l'enseignement du FLE dépassent les limites indéniablement inhérentes aux jeux de rôles. Ces derniers comprennent des scénarios souvent trop figés et engendrant, de fait, une parole des apprenants trop contrainte, sans réflexion approfondie. C'est pourquoi, nous incitons vivement les enseignants qui souhaitent bénéficier des apports de l'improvisation à mettre en place des séances de théâtre-forum. Pour cela, ils peuvent se baser sur les étapes d'un atelier de théâtre-forum que nous précisons en détail dans la dernière section de notre article.

\section{Notes}

1 Dans cet article, le théâtre-forum est considéré comme un outil permettant le développement de la compétence interculturelle dans sa dimension émotionnelle et sociale chez des individus (enseignants ou apprenants) évoluant dans des zones frontalières européennes. Même s'ils considèrent l'autre (celui vivant de l'autre côté de la frontière) comme relativement proches d'eux, ces sujets peuvent, au regard de leurs histoires personnelles, le rejeter ou avoir des représentations négatives sur sa langue, qu'ils sont souvent obligés d'apprendre dans des parcours de formation bilingues. Le théâtre-forum leur permet de modifier leurs représentations.

\section{Bibliographie}

Boal, Augusto. «Le flic dans la tête ou les trois hypothèses. » Le théâtre d'intervention depuis 1968 : Tome II. Lausanne : L'âge d'homme, 1983. 165-172. Imprimé.

---. Jeux pour acteurs et non-acteurs : pratique du Théâtre de l'opprimé. Paris : La Découverte, 2004. Imprimé. 
Del Olmo, Claire. « Improviser en classe de Français Langue Étrangère. » Nouvelle Revue Synergies Canada, N8 (2015)

---. The Aesthetics of the Oppressed. New York : Routledge, 2006. Imprimé.

Bordet, Joëlle. Oui à une société avec les jeunes des cités ! Sortir de la spirale sécuritaire. Paris : Editions de l'Atelier/Editions Ouvrières, 2007. Imprimé.

Breugnot, Jacqueline. "La formation interculturelle face à la diversité culturelle. » Actes du VIIlème Congrès de l'Association pour la Recherche Interculturelle (ARIC) Université de Genève. 24-28 septembre 2001. Web. 20 octobre 2014.

---. « La compréhension interculturelle en zone frontalière: quels besoins, quels outils, quelles formations ? » Penser le bilinguisme autrement. Frankfurt : Peter Lang, 2008. 97-110. Imprimé.

Cuq, Jean Pierre. Éd. Dictionnaire de didactique du français langue étrangère et seconde. Paris: CLE International, 2003. Imprimé.

Evers, Angela. Le grand livre de l'art-thérapie. Paris : Eyrolles, 2010. Imprimé.

Guerre, Yves. Le théâtre-forum : pour une pédagogie de la citoyenneté. Paris : L'Harmattan, 1999. Imprimé.

Leblanc, Marie Cécile. Jeu de rôle et engagement : évaluation de l'interaction dans les jeux de rôles de français langue étrangère. Paris : L'Harmattan, 2002. Imprimé.

Lemeunier, Valérie. «Le marché du FLE : un marché de dupes ? » Une identité plurielle : mélanges offerts à Louis Porcher. Paris : L'Harmattan, 2003. 355-368. Imprimé.

d'Orgeval, Martine. «Les styles d'animation. » Guide de la formation et du développement professionnel. 2e éd. Paris : Retz, 2006. Imprimé.

Pauzet, Anne. « Un théâtre-forum pour apprendre la rencontre interculturelle. » Les Langues Modernes 2.24 juin 2010. Web. 20 octobre 2014.

Puren, Christian. « De l'approche communicative à la perspective actionnelle. » Le Français dans le monde 347. Septembre-octobre (2006) : 37-40. Imprimé.

Ramat, Jean Paul. « Faire du théâtre hors du théâtre. » Les usages sociaux du théâtre hors ses murs: école, entreprise, hôpital, prison, etc. Paris : L'Harmattan, 2011. Imprimé.

Robert, Jean Pierre. Dictionnaire pratique de didactique du FLE. Paris : Editions Ophrys, 2008. Imprimé. 